\title{
Thoracoscopic off-pump closure of a large left circumflex coronary artery fistula: A novel minimally invasive approach
} \author{
Brussels, Belgium \\ From the Departments of ${ }^{\mathrm{a}}$ Cardiac Surgery, ${ }^{\mathrm{b}}$ Cardiology, and ${ }^{\mathrm{c}}$ Radiology, Universitair Ziekenhuis Brussel \\ Brussels, Belgium. \\ Disclosures: Authors have nothing to disclose with regard to commercial support. \\ Received for publication Feb 26, 2018; revisions received March 27, 2018; accepted for publication April 1, 2018 ; \\ available ahead of print May 11, 2018. \\ Address for reprints: Ines Van Loo, MD, Department of Cardiac Surgery, Universitair Ziekenhuis Brussel, \\ Laarbeeklaan 101, 1090 Jette, Belgium (E-mail: ines.vanloo@uzbrussel.be). \\ J Thorac Cardiovasc Surg 2018;156:e159-61 \\ $0022-5223 / \$ 36.00$ \\ Copyright (c) 2018 by The American Association for Thoracic Surgery \\ https://doi.org/10.1016/j.jtcvs.2018.04.041
}

Ines Van Loo, MD, ${ }^{\mathrm{a}}$ Jeroen Sonck, MD, ${ }^{\mathrm{b}}$ Kaoru Tanaka, MD, PhD, ${ }^{\mathrm{c}}$ and Mark La Meir, MD, PhD, ${ }^{\mathrm{a}}$ $\triangleright$

Video clip is available online.

A coronary artery fistula (CAF) is an uncommon anomalous communication between a coronary artery and a cardiac chamber, the coronary sinus, the caval veins, the pulmonary artery, or a pulmonary vein. ${ }^{1}$ It can result in a coronary steal phenomenon and myocardial ischemia. A chronic left-to-right shunt can lead to pulmonary arterial hypertension and congestive heart failure. Rare presentations are endocarditis, arrhythmia, and cardiac tamponade. Most patients, however, remain free of symptoms. ${ }^{2}$

Surgical closure of CAF through sternotomy or thoracotomy has been described as safe and effective. ${ }^{1-3}$ Such closure often requires cardiopulmonary bypass (CPB) to allow visualization of a proximal origin or to identify the CAF course while maintaining hemodynamic stability. ${ }^{3}$ Percutaneous closure is a less invasive alternative with comparable closing results. ${ }^{4}$ In a case of CAF with anatomy less suited for endovascular closure, a thoracoscopic approach could offer an alternative.

\section{CLINICAL SUMMARY}

A 51-year-old man was admitted with a new onset of dyspnea and peripheral edema. He also had retrosternal pain during cycling. A recurrent left pleural effusion was investigated with a computed tomographic scan, which revealed a large fistula between the proximal left circumflex coronary artery (LCX) and the superior vena cava. Cardiac catheterization confirmed the CAF. An important steal phenomenon and pulmonary arterial hypertension were found.

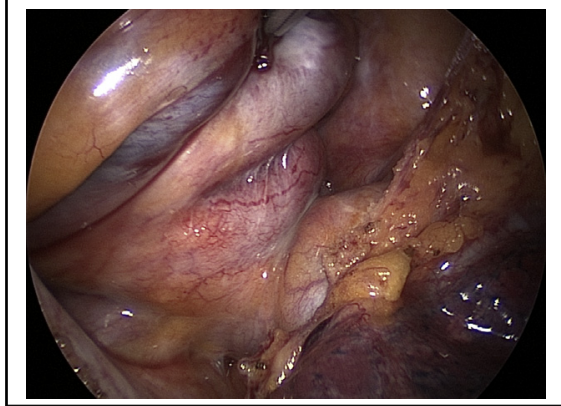

Excellent visualization of the LCX fistula through left-sided thoracoscopic approach.

\section{Central Message}

This is the first article to report the off-pump thoracoscopic approach for the treatment of coronary artery fistula. No complications occurred. Follow-up showed no residual flow or recurrence.

See Editorial Commentary page e163
Percutaneous closure was ruled out because of the CAF's large diameter throughout its entire course and because of a high probability to compromise the LCX. Since the proximal part of the LCX is difficult to reach through sternotomy, possibly requiring $\mathrm{CPB}$ and transection of the aorta, we proposed a thoracoscopic procedure.

The intervention is shown in Video 1. The patient was draped from neck to groin. A left thoracoscopy was performed by an experienced thoracoscopic cardiac surgeon (M.L.M.) with 3 5-mm ports and 1 10-mm port. CPB was standby if needed for central or femoral cannulation. A cell saver was prepared to recover any significant blood loss, which did not occur. In the event of major bleeding, we would have converted to sternotomy. The pericardium was opened below the phrenic nerve, and the fistula was prepared to achieve hemostatic control proximal and distal from the predefined proximal stapler line. With a $30-\mathrm{mm}$ vascular stapler (Endo GIA Tri-Staple; Covidien, Ireland) complete transection of the CAF was achieved 5-mm from its origin. Transection of the distal end at the superior vena cava was not performed because the low pressure in 


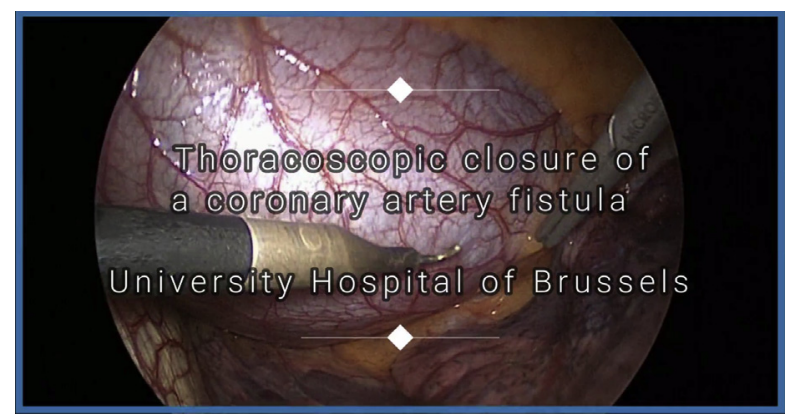

VIDEO 1. Through a left thoracoscopy, the pericardium was opened below the phrenic nerve, which resulted in an excellent visualization of the left circumflex coronary artery fistula. After careful dissection, the fistula was transected with a 30-mm vascular stapler, 5-mm from its origin at the left circumflex coronary artery. Video available at: https://www.jtcvs. org/article/S0022-5223(18)31022-5/fulltext.

this system was anticipated to lead to spontaneous obliteration.

The patient was discharged at day 5 . He was treated for pericarditis. No other complications occurred. The computed tomographic scan after 2 months showed a successful closure, with a small, predefined remainder of $0.5 \mathrm{~cm}$ (Figure 1). At 5-month follow-up, the patient was free of symptoms.

\section{DISCUSSION}

A fistula originating from the LCX is rare. In the largest surgical series, reported by Hou and colleagues, ${ }^{1}$ all 29 LCX CAFs were treated through sternotomy, with only 4 of the 29 performed off pump. In the series of Cheung and associates $^{2}$ and Said and coworkers ${ }^{5}$ concerning all types of CAF, CPB was needed in 23 of 41 and 39 of 46 patients, respectively. The decision to use CPB was often made because of a proximal CAF origin or because of concomitant cardiac disease. ${ }^{5}$ Zhang and colleagues ${ }^{3}$ reported a $91.5 \%$ use of $\mathrm{CPB}$ in their surgical series of pediatric $\mathrm{CAF}$.

In isolated CAF, open chest surgery can be avoided by percutaneous techniques with coils, occluding devices, or covered stents in case of atherosclerotic disease. ${ }^{4,5}$ Although less invasive, percutaneous closure carries potential complications, such as device migration, entrapment of a guidewire, or dissection. ${ }^{3}$ Coronary tortuosity, adjacent vessels at risk, very large fistulas, multiple communications, or the absence of a narrow ending are possible contraindications for transcatheter closure. $^{3,4}$ In such cases, a thoracoscopic approach could be an alternative. The general advantages of thoracoscopy versus sternotomy include less postoperative pain, shorter hospital stay, decreased inflammatory cytokine response, less blood loss, and superior cosmetic result. Especially for the LCX CAF, a thoracoscopic approach has the advantage of proper visualization without the need to tilting the heart or transect any structures, obviating the need for CPB.

Recanalization and incomplete occlusion with residual shunt are reported as many as $20 \%$ of cases in surgical as well as percutaneous reports. ${ }^{1,3,5}$ This complication did not occur in our case; however, longer follow-up is necessary.
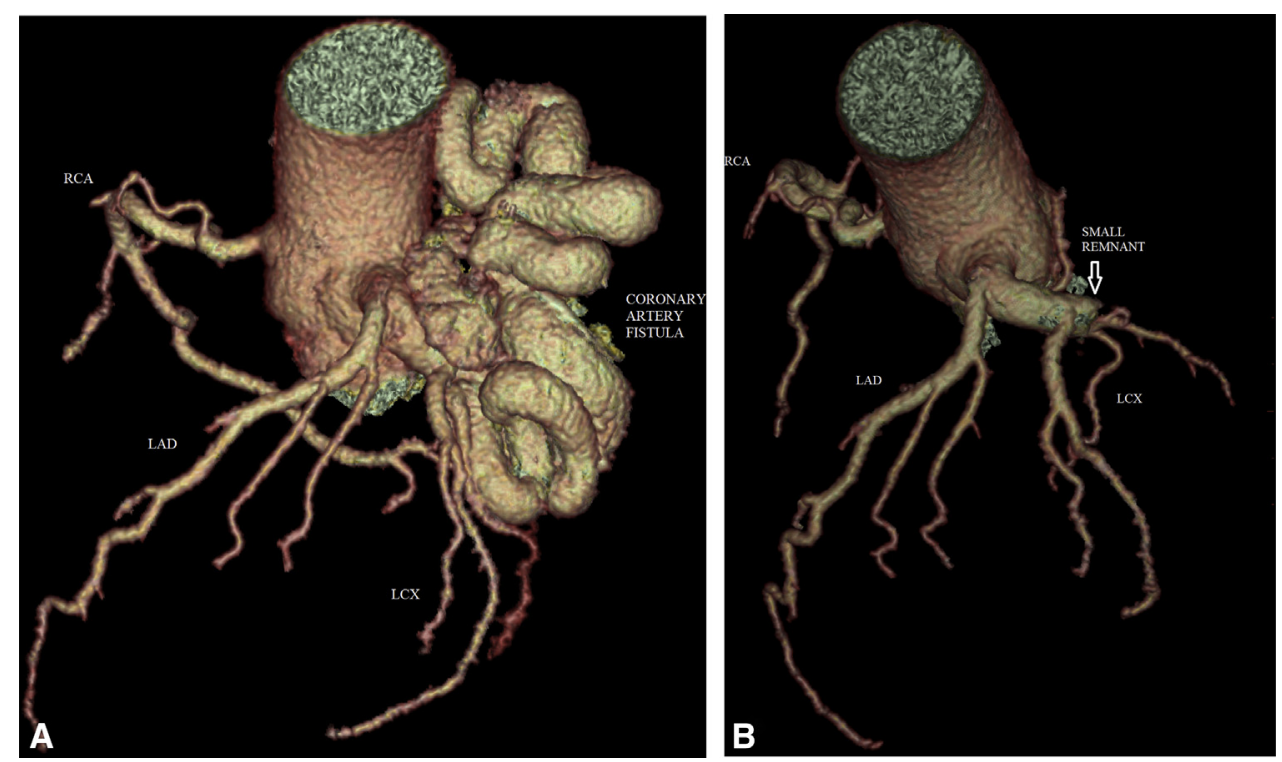

FIGURE 1. Computed tomographic imaging. A, Preoperative coronary computed tomographic angiogram showing a large fistula, originating from the proximal left circumflex coronary artery $(L C X)$ and ending in the superior vena cava (not shown). B, Postoperative imaging after 2 months, showing a successful closure with a small remnant of $0.5 \mathrm{~cm}$. RCA, Right coronary artery; $L A D$, left anterior descending artery. 
We conclude that the thoracoscopic transection of LCX CAF is feasible, safe, and effective in the hands of an experienced thoracoscopic surgeon. The operating team should be capable of handling bleeding, ventricular fibrillation, hemodynamic instability, and ischemia.

\section{References}

1. Hou B, Ma W, Zhang J, Du M, Sun H, Xu J, et al. Surgical management of left circumflex coronary artery fistula: a 25-year single-center experience in 29 patients. Ann Thorac Surg. 2014;97:530-6.
2. Cheung DL, Au WK, Cheung HH, Chiu CS, Lee WT. Coronary artery fistulas: long-term results of surgical correction. Ann Thorac Surg. 2001;71: $190-5$.

3. Zhang W, Hu R, Zhang L, Zhu H, Zang H. Outcomes of surgical repair of pediatric coronary artery fistulas. J Thorac Cardiovasc Surg. 2016;152: 1123-30.e1.

4. Okubo M, Nykanen D, Benson LN. Outcomes of transcatheter embolization in the treatment of coronary artery fistulas. Cathet Cardiovasc Interv. 2001; 52:510-7.

5. Said SM, Burkhart HM, Schaff HV, Connolly HM, Phillips SD, Suri RM, et al, Late outcome of repair of congenital coronary artery fistulas - a word of caution. J Thorac Cardiovasc Surg. 2013;145:455-60. 